\title{
ON DEVELOPABLE AND TUBULAR SURFACES HAVING SPHERICAL LINES OF CURVA- TURE.
}

BY PROFESSOR VIRGIL SNYDER.

(Read before the American Mathematical Society, April 30, 1904.)

IT has been shown by Peters* that if the asymptotic lines of the second system on a scroll belong to linear complexes, the scroll must have two rectilinear directrices. This theorem, interpreted in spherical space becomes: If an annular surface has plane or spherical lines of curvature of the second system, the spheres or planes must all belong to a pencil.

In particular, if the theorem be applied to developable surfaces, the generating planes of the latter must belong to a linear spherical complex

$$
\psi \equiv a \xi+b \eta+c \xi+d \lambda+e \mu+f \nu=0
$$

as well as to the complex of planes

$$
\Pi \equiv{ }^{*} \mu-\nu=0 .
$$

The generating planes must touch the directrices of this congruence, which are two concentric spheres; or, if $e+f=0$, the fundamental sphere of every complex of the pencil becomes a plane, and these planes are parallel.

The necessary and sufficient condition that the planes belong to a complex $E(e+f=0)$ is that the variable coefficients $u(t), v(t), w(t), s(t)$ in the equation of the generating plane

$$
u x+v y+w z+s=0
$$

satisfy a relation of the form

$$
A u+B v+C w=D \sqrt{u^{2}+v^{2}+w^{2}} \sqrt{A^{2}+B^{2}+C^{2}} .
$$

\footnotetext{
* "Die Flächen, deren Haupttangentenkurven linearen Complexen angehören," Christiania [Leipzig dissertation, 1895].
} 
Let the plane containing a line of curvature be chosen for the (x,y)-plane. $A=0, B=0$, so that the relation becomes

$$
u^{2}+v^{2}=l w^{2}
$$

The equation of the plane may be written

Let

$$
z+\frac{u}{w} x+\frac{v}{w} y+\frac{s}{w}=0
$$

$$
u / w=\tau, \quad v / w=\sqrt{l-\tau^{2}} ;
$$

then the equation of the generating plane becomes

$$
z+\tau y+\sqrt{l-\tau^{2}} x+\phi(\tau)=0,
$$

in which $\phi(\tau)$ is an arbitrary function of $\tau$.

If the curved lines of curvature of a developable surface are plane, the planes are parallel. If these planes be of the form $z=c$, the developable is the envelope of the plane

$$
z+\sqrt{l-\tau^{2}} x+\tau y+\phi(\tau)=0 .
$$

Let the parameter $\tau$ and the function $\phi(\tau)$ be replaced by a new parameter $k$ and a function $f(k)$ defined by the equation

$$
\phi(\tau)=k \tau+f(k) \sqrt{l-\tau^{2}} .
$$

No restriction is placed upon the old or the new function by this substitution. Differentiate as to $\tau$,

$$
\phi^{\prime}(\tau)=k-\frac{\tau f(k)}{\sqrt{l-\tau^{2}}}
$$

and substitute the result in the derivative of the generating plane,

Thus

$$
0=\frac{-\tau}{\sqrt{l-\tau^{2}}} x+y+\phi^{\prime}(\tau)
$$

from which

$$
\frac{\tau}{\sqrt{l-\tau^{2}}} x-y=k-f(k) \frac{\tau}{\sqrt{l-\tau^{2}}},
$$




$$
(k+y)^{2}=\frac{\tau^{2}}{l-\tau^{2}}(f(k)+x)^{2},
$$

or, solving for $\tau$,

$$
\begin{aligned}
& \boldsymbol{\tau}=\frac{\sqrt{l}(y+k)}{\sqrt{(x+f(k))^{2}+(y+k)^{2}}}, \\
& \sqrt{l-\tau^{2}}=\frac{\sqrt{l}(x+f(k))}{v^{\prime}(x+f(k))^{2}+(y+k)^{2}} .
\end{aligned}
$$

These values are now to be substituted in the equation of the generating plane

$$
z+\sqrt{l-\tau^{2}} x+\tau y+\phi(\tau)=0 .
$$

The result may be written

$$
z^{2}=l\left[(x+f(k))^{2}+(y+k)^{2}\right] .
$$

The surface is thus seen to be the envelope of a right circular cone of constant angle, whose axis is always perpendicular to the plane lines of curvature, but whose vertex describes an arbitrary plane curve. The plane sections $z=c$ are seen also to be the envelope of a circle of fixed radius $c$ whose center describes the curve

$$
a=-f(k), \quad \beta=-k ;
$$

hence the lines of curvature in the planes $z=c$ project orthogonally into a system of parallel curves.

Minimum developables and the developables whose cuspidal edges are general helices are included in this category; but others whose cuspidal edges are not helices are also included.

Similarly, if the lines of curvature are spherical the spheres must be concentric. All of the generating planes are tangent to a sphere along any arbitrary curve. If the planes all pass through a point the surface reduces to a cone.

If the center of the family of concentric spheres is taken as origin, the parameter $\tau$ of the generating plane must satisfy the relation

$$
s^{2}=r^{2}\left(u^{2}+v^{2}+w^{2}\right)
$$

so that here two coördinates are arbitrary. The equation of the plane may be written 
or also

$$
\tau x+\phi(\tau) y+\sqrt{k^{2}-\tau^{2}-\phi(\tau)} z=1 ;
$$

$$
V \overline{[k \phi(\tau)]^{2}}-1-\tau^{2} x+\tau y+z=\phi(\tau) .
$$

A tubular surface is the envelope of a sphere of constant radius whose center describes an arbitrary curve. If the lines of curvature of such a surface are to be plane or spherical, the surface must be contained in the congruence

$$
\begin{gathered}
d^{\prime} \lambda+e^{\prime}(\mu-\nu)=0, \\
\psi \equiv a \xi+b \eta+c \zeta+d \lambda+e \mu+f \nu=0 .
\end{gathered}
$$

The directrices of this congruence are two spheres concentric with the fundamental sphere of $\psi=0$, and the locus of centers is therefore a sphere. Hence when the lines of curvature of a tubular surface are spherical, the locus of centers must be a spherical curve. The curves of intersection made with the surface by the pencil of spheres concentric with that of the locus of centers are their lines of curvature.

If in $\psi e+f=0$, the fundamental sphere becomes a plane. The directrices are in this case two parallel planes and the locus of centers becomes a plane curve. The lines of curvature are now in a series of parallel planes, as is also geometrically evident.

The theorem regarding developables is thus at once applicable to tubular surfaces.

The section made by the plane at infinity with any tubular or developable surface is always a line of curvature. If the surface contains one other plane (or spherical) line of curvature, then all lines of the system must also be plane (spherical). For a treatment of the general annular surface having spherical lines of curvature see two papers by the author in the American Journal of Mathematics, volumes 22 and 23.

When a surface has one system of lines of curvature, lying in parallel curves, the other system is plane also and the projection of the lines of both systems upon a plane parallel to the former will be an orthogonal system. The converse is also true, namely :

Surfaces whose lines of curvature of both systems are orthogonally projected into an orthogonal system on a plane are surfaces having one system of lines of curvature lying in parallel planes. 
Since then all the lines of curvature of both systems belong to spherical complexes whose fundamental spheres become planes, and the planes of one system cut both the surface and the planes of the parallel system orthogonally, the parallel planes are fundamental spheres of a system of complexes of the form

$$
c \zeta+d \lambda+e(\mu-\nu)=0
$$

in which without loss of generality $c=1, d=\tau, e=f(\tau)$. In particular, if $f(\tau)$ is a linear function, the surface is either a tube or a developable.

In the general case, the other system of lines of curvature belong to the complexes

$$
a \xi+b \eta-h(\mu-\nu)=0,
$$

in which $a=1, b=k, h=\phi(k)$. If $f(\tau)$ is not linear, $\phi(k)$ cannot be linear.

The first system of complexes have in common one set of generators of a Dupin's cyclide. The second system contains the other set of generators.

The discriminant (invariant) of the characteristic cyclide vanishes, but not all of the first minors vanish. The cyclide therefore reduces to a gauche quadrilateral of minimum lines. This same property will also hold for every surface into which the surface may be transformed by inversion in a linear complex.

The lines of curvature of any inversion of a moulded surface are spherical in both systems, and the characteristic Dupin's cyclide degenerates into a gauche quadrilateral of minimum lines.

When all the lines of curvature of both systems are plane, the characteristic Dupin cyclide must consist of planes in both generations. It was shown by Bonnet that the planes containing the lines of curvature of one system are tangent to a cylinder, and those of the other system tangent to another cylinder whose axis is perpendicular to that of the first.

If $x=0, y=0$ be the equations of the first axis, the net of complexes to which the lines of curvature of the first system belong may be written in the form

$$
E+\theta E^{\prime}+t E^{\prime \prime}=0
$$


where

and

$$
E^{i} \equiv a^{i} \xi+b^{i} \eta+d^{i} \lambda+e^{i}(\mu-\nu)
$$

$$
\Sigma a a^{\prime}=0, \quad \Sigma a a^{\prime \prime}=0, \quad \Sigma a^{\prime} a^{\prime \prime}=0 .
$$

Similarly, if $x=0, z=0$ be the second axis, we have

with

$$
E_{1}+\theta_{1} E_{1}{ }^{\prime}+f_{1} E_{1}^{\prime \prime}=0,
$$

$$
\Sigma a_{1} a_{1}{ }^{\prime}=0, \quad \Sigma a_{1} a_{1}{ }^{\prime \prime}=0, \quad \Sigma a_{1} a_{1}{ }^{\prime \prime}=0 .
$$

Moreover, every complex of the first system must be in involution with every complex of the second. These results show that the invariant of one of the complexes of each system vanishes. Since the discriminant of a series is invariant under linear transformation of the hexaspherical coördinates, surfaces having only plane lines of curvature can not be transformed into general surfaces having spherical lines of curvature by inversion in a linear complex. This configuration is treated in my dissertation, $\S 18$, III, $1 \beta$, page 45 . The cyclide becomes a quadrilateral inscribed in the absolute circle at infinity.

Cornele University,

April, 1904.

\section{ADDITION TO A THEOREM DUE TO FROBENIUS.}

BY PROFESSOR G. A. MILLER.

(Read before the San Francisco Section of the American Mathematical Society, April 30,1904.)

IN a paper presented by the writer at a recent meeting of the London mathematical society it is proved that the number of cyclic subgroups of order $p^{s}, p$ being any odd prime and $s>1$, which are contained in a non-cyclic group of order $p^{m}$ is always a multiple of $p$. From this theorem it follows directly that the number of cyclic subgroups of order $p^{s}$ in any group $G$ must be a multiple of $p$ whenever the Sylow subgroups of order $p^{m}$ are non-cyclic. When these Sylow subgroups are cyclic the number of the cyclic subgroups of order $p^{s}$ contained in $G$ is clearly of the form $1+\mathrm{kp}$. In all other cases $G$ contains $1+k p$ non-cyclic subgroups of order $p^{s}$. In other words, if $G$ 